\title{
D-dimer is Valuable for Predicting the Extension of Isolated Distal Deep Vein Thrombosis of the Lower Limb in Neurocritical Patients: A Retrospective Observational Study
}

\section{Rui Tang ( $\sim$ drtangrui@126.com )}

The First Affiliated Hospital of USTC: Anhui Provincial Hospital https://orcid.org/0000-0002-6110-3578

\section{Zhi Gao}

The First Affiliated Hospital of USTC: Anhui Provincial Hospital

\section{Min Du}

The First Affiliated Hospital of USTC: Anhui Provincial Hospital Haiyan Liu

The First Affiliated Hospital of USTC: Anhui Provincial Hospital

\section{Yanyan Yang}

The First Affiliated Hospital of USTC: Anhui Provincial Hospital

\section{Zongsu Ji}

The First Affiliated Hospital of USTC: Anhui Provincial Hospital

\section{Min Zhou}

The First Affiliated Hospital of USTC: Anhui Provincial Hospital

\section{Research}

Keywords: Acute brain injury, Isolated distal deep vein thrombosis, Coagulation function

Posted Date: April 19th, 2021

DOI: https://doi.org/10.21203/rs.3.rs-420412/v1

License: (c) (1) This work is licensed under a Creative Commons Attribution 4.0 International License. Read Full License 


\section{Abstract}

Background: Isolated distal deep vein thrombosis (IDDVT) of the lower limb and its extension to the proximal deep veins have high incidence rates in patients with acute brain injury (ABI). The objective of the study was to determine the value of dynamic changes in coagulation function in predicting the occurrence and extension of IDDVT.

Methods: In this retrospective cohort study, $A B I$ patients during the perioperative period in a neurocritical care unit (NICU) of a university hospital were identified from September 2019 to September 2020. Complete compression ultrasound was used by a senior sonographer to diagnose the occurrence and extension of IDDVT. Coagulation function was recorded at a series of time points during the perioperative period.

Results: A total of $245 \mathrm{ABI}$ patients were identified, including 46 acute traumatic brain injury patients, 117 acute hemorrhagic stroke patients, 36 acute ischemic stroke patients and 46 acute aneurysmal subarachnoid hemorrhage patients. Most of the patients were elderly and male, and most had severe cases. The rates of IDDVT occurrence and extension were $62 \%$ and $21 \%$, respectively. Age, GCS score at admission, NICU length of stay were risk factors for IDDVT occurrence. None of coagulation indices was a sensitive predictor of IDDVT occurrence. The elevation of D-dimer on days 5-7 was the most valuable predictor of IDDVT extension (the area under the ROC was 0.89 , with a $95 \% \mathrm{Cl}$ of $0.82-0.95$, and the sensitivity and specificity were 0.86 and 0.81 , respectively with the cutoff of $8.9 \mathrm{mg} / \mathrm{L}$ ).

Conclusions: The occurrence and extension of IDDVT are common in ABI patients during the perioperative period, particularly in elderly patients with severe cases. D-dimer level on days 5-7 is the most valuable predictor of IDDVT extension in ABI patients.

\section{Introduction}

Acute brain injury $(\mathrm{ABI})$ patients (including acute traumatic brain injury, acute hemorrhagic stroke, acute ischemic stroke and acute aneurysmal subarachnoid hemorrhage patients) who are treated in the neurocritical care unit are at high risk for deep vein thrombosis (DVT) of the lower limb; the risk varies considerably from 17-40\% with different diagnostic strategies[1-4]. Isolated distal deep vein thrombosis (IDDVT) of the lower limb, also known as calf DVT, refers to infrapopliteal vein DVT that does not extend to proximal veins[5]. IDDVT is a very common medical condition, accounting for up to $50 \%$ of lower limb DVT[6]. In neurocritical care patients, the proportion of IDDVT is as high as 76\%[2].

The extension of IDDVT to proximal veins and the associated risk of pulmonary embolism (PE) are focal points in the management of IDDVT[6]. In low-risk patients, it may be safe not to administer anticoagulation due to the low rate of IDDVT extension and PE. However, for high-risk patients, such as ABI patients, the optimal management of IDDVT is still uncertain. The frequency of IDDVT extension in high-risk patients has been reported to reach nearly $17 \%$ [7]. 
Complete compression ultrasound (CCUS), investigating both distal and proximal veins, is currently the main diagnostic strategy for IDDVT and its extension [8,9]. CCUS has lower sensitivity and specificity than two-point compression ultrasound for proximal DVT. A meta-analysis showed that the sensitivity and specificity of CCUS for IDDVT were $60-67 \%$ and $93-94 \%$, respectively[10]. Other shortcomings of CCUS include the need for highly skilled ultrasonographers and sophisticated ultrasound machines, high rates of inconclusive results and high fees for repeated diagnostic tests. A recent study by Hargrove et al. demonstrated that compared with a symptom-driven diagnostic protocol, routine surveillance ultrasound did not significantly reduce the time to DVT diagnosis in neurocritical care patients [11]. Hence, the selection of $A B I$ patients to undergo CCUS is a dilemma to intensivists.

The objective of this study was to elucidate the characteristics of and risk factors for the occurrence and extension of IDDVT in ABI patients and to determine the value of dynamic changes in coagulation function for predicting these IDDVT-related events.

\section{Methods}

\section{Study Design and Population}

The study was a single-center retrospective cohort study performed in a 28-bed neurointensive care unit (NICU) of a university hospital in China. The Ethics Committee of our hospital approved the study and waived the requirement for informed consent because of the minimal risk to participants.

Using an electronic patient data recording system, all patients admitted to our NICU from September 2019 to September 2020 were screened. The inclusion criteria comprised ABI patients transferred to the NICU within $24 \mathrm{~h}$ after surgery or endovascular therapy (including acute traumatic brain injury, acute hemorrhagic stroke, acute ischemic stroke and acute aneurysmal subarachnoid hemorrhage). Patients were excluded if they had any of the following characteristics: age $<18$ years; pregnancy at the time of hospitalization; length of NICU stay < 3 days; therapeutic anticoagulation already in progress before transfer to the NICU; previously diagnosed DVT; active tumor; severe liver or kidney disease; long-term bedridden status; or lack of CCUS scans.

All patients were treated according to guidelines and received DVT prevention measures, including intermittent pneumatic compression and passive limb exercises, as soon as possible. The use of chemical DVT prophylaxis or treatment via low-molecular-weight heparin, heparin, etc., was determined by the attending doctor.

\section{Patient Data Collection}

A standardized sheet was used by investigators (Z. G. and R. T.) to collect information from patients' electronic medical records. Data included baseline characteristics (age, sex, height, weight, and diagnosis); disease history (cardiovascular, pulmonary, hepatic, renal, diabetes); risk factors for DVT (current smoking, estrogen use, ambulatory status, family history of hypercoagulability); disease 
characteristics (disease onset time; GCS score at admission; APACHE II score at transfer in; brain herniation; comorbidities during treatment, such as shock, hepatic injury, renal injury, gastrointestinal bleeding, sepsis, or pulmonary embolus); treatment approaches (surgery or endovascular therapy; operation time; decompressive craniectomy; second surgery; mechanical ventilation; renal-replacement therapy; transfusion, such as red blood cells, plasma, platelets, anticoagulation therapy and its onset time); and patient outcomes (fatality in the NICU, length of NICU stay, GCS score at transfer out).

\section{CCUS procedures}

All CCUS scans were performed according to the guidelines for ultrasound for lower extremity DVT [12] and were carried out by experienced sonographers (C.T., M.Y., and X.H.) who were not aware of the patients' clinical features or coagulation test results. A color duplex ultrasound system (Vivid iq: GE Healthcare, Milwaukee, USA) with a 5 to $10 \mathrm{MHz}$ transducer was used for the scans. CCUS was performed once or twice weekly during each patient's NICU stay.

\section{Coagulation tests}

Coagulation tests, including prothrombin time (PT), activated partial thromboplastin time (APTT), fibrinogen, thrombin time (TT) and D-dimer, based on clotting (viscosity-based detection system), chromogenic and immunologic assays were performed in all patients at The First Affiliated Hospital of USTC Central Clinical Chemistry Laboratory (The STA R Max®, Diagnostica Stago, Asnières, France). All

tests were performed on the first day and at a series of later timepoints (once or twice weekly) during the NICU stay.

\section{Statistical analysis}

Normally distributed data are presented as the means \pm standard deviations. Other continuous data are expressed as medians and interquartile ranges. Categorical variables were expressed as $\mathrm{n}(\%)$. Student's t-tests, Mann-Whitney U tests, chi-square tests or Fisher's exact tests were used to compare variables between different groups. A multivariate analysis was performed using logistic regression models with a stepwise approach according to univariate results $(P<0.10)$ and clinical relevance. The results are expressed as odds ratios (ORs) and $95 \%$ Cls. Log likelihood was used to measure the goodness of fit of each model.

The effects of IDDVT and time on the measured parameters were examined by two-way repeatedmeasures ANOVA, followed by a Bonferroni correction for multiple comparisons. In order to simplify statistical analysis, the data from the first day in the NICU were labeled day 1 , the data from the second through fourth days were labeled days $2-4$, the data from the fifth through seventh days were labeled days $5-7$, and the data beyond the seventh day were labeled day $>7$.

For each parameter that differed between groups, a receiver operating characteristic (ROC) curve was plotted to identify optimal predictors of IDDVT and its extension by utilizing the area under the curve 
(AUC). The threshold values of the parameters were determined according to usual recommendations by estimating several indexes such as the Youden index.

$P<0.05$ was considered statistically significant. SPSS 26.0 (SPSS, Chicago, USA) was used for statistical analysis.

\section{Results}

During the study period, 814 patients were admitted to our NICU. Of these admitted patients, 254 were enrolled (Fig. 1).

\subsection{The characteristics of the included ABI patients (Table 1)}


Table 1

The characteristic of acute brain injury patients included

\begin{tabular}{|l|l|}
\hline Variables & Statistical data \\
\hline Number (n) & 245 \\
\hline Male (n/\%) & $142(58 \%)$ \\
\hline Age (year) & $62(52-69)$ \\
\hline Diagnosis (n/\%) & \\
\hline acute traumatic brain injury & $46(18.8)$ \\
\hline acute hemorrhagic stroke & $117(47.8)$ \\
\hline acute ischemic stroke & $36(14.7)$ \\
\hline acute aneurysmal SAH & $46(18.8)$ \\
\hline GCS on admission & $6(5-8)$ \\
\hline APACHE II score at transfer in & $23(21-25)$ \\
\hline The length of ICU stay (days) & $13(8-17)$ \\
\hline GCS at transfer out & $7(5-8)$ \\
\hline The incidence of DVT (n/\%) & $157(64.1)$ \\
\hline Proximal DVT & $28(11.4)$ \\
\hline IDDVT & $151(61.6)$ \\
\hline Asymptomatic DVT & $126(80.3)$ \\
\hline IDDVT extension & $22(20.8)$ \\
\hline Anticoagulation treatment (n/\%) & $92(37.6)$ \\
\hline Acute pulmonary embolus (n/\%) & $1(0.6)$ \\
\hline NICU mortality (n/\%) & $18(7.3)$ \\
\hline not normally distributed. GCS, Glasgow Coma Scale; APACHE II score, the acute physiology and \\
thrombosis.
\end{tabular}

The ABI patients included were mainly male (58\%) and elderly (median age, 62 years), and their brain injury tended to be severe (median GCS score at admission, 6; median APACHE II score at transfer in, 23). Acute hemorrhagic stroke was the most common disease (47.8\%), followed by aSAH (18.8\%), acute traumatic brain injury (18.8\%), and acute ischemic stroke (14.7\%). The median length of NICU stay was 13 days, the median GCS score at transfer out was 7, and the mortality of NICU was $7.3 \%$. The percentage 
of patients receiving anticoagulation treatment in NICU was $37.6 \%$. The median starting time for anticoagulant therapy was the eighth day.

The incidence of lower limb DVT during NICU stay was $64.1 \%$. The incidence of IDDVT and proximal DVT were $61.6 \%$ and $11.4 \%$ respectively. Most of the lower limb DVT was asymptomatic DVT (80.3\%). The incidence of IDDVT extension was $20.8 \%$. The median time for the first diagnosis of IDDVT occurrence and extension were the fourth and ninth day, respectively. Only 1 acute pulmonary embolus occurred among the included patients, and it was deemed a low-risk PE.

3.2 The characteristics of IDDVT occurrence and extension and the associated risk factors (Table 2-4)

Table 2

Demographic data, clinical data of patients with IDDVT occurrence or extension compared with control subjects

\begin{tabular}{|c|c|c|c|c|c|c|c|c|}
\hline & \multicolumn{2}{|c|}{ IDDVT occurrence } & \multicolumn{2}{|c|}{ Statistical data } & \multicolumn{2}{|c|}{ IDDVT extension } & \multicolumn{2}{|c|}{ Statistical data } \\
\hline & $\begin{array}{l}\text { Yes }(n= \\
129)\end{array}$ & $\begin{array}{l}\text { No }(n= \\
88)\end{array}$ & $t / x$ & $p$ & $\begin{array}{l}\text { Yes }(n= \\
22)\end{array}$ & $\begin{array}{l}\text { No }(n= \\
84)\end{array}$ & $t / x$ & $p$ \\
\hline Age (years) & $62 \pm 12$ & $57 \pm 14$ & -2.998 & 0.003 & $61 \pm 17$ & $61 \pm 13$ & -0.035 & 0.973 \\
\hline Male (n/\%) & $65(50.4)$ & $59(67.0)$ & 5.927 & 0.015 & $15(68.2)$ & $47(56.0)$ & 1.074 & 0.300 \\
\hline $\mathrm{BMI}\left(\mathrm{kg} / \mathrm{m}^{2}\right)$ & $21 \pm 7$ & $23 \pm 4$ & 3.167 & 0.002 & $21 \pm 7$ & $22 \pm 6$ & 0.550 & 0.587 \\
\hline $\begin{array}{l}\text { GCS on } \\
\text { admission }\end{array}$ & $6 \pm 2$ & $7 \pm 2$ & 2.829 & 0.005 & $6 \pm 2$ & $6 \pm 2$ & -0.057 & 0.955 \\
\hline $\begin{array}{l}\text { APACHE II score } \\
\text { at transfer in }\end{array}$ & $23 \pm 3$ & $23 \pm 4$ & 0.029 & 0.977 & $24 \pm 4$ & $23 \pm 3$ & -0.941 & 0.354 \\
\hline $\begin{array}{l}\text { Brain herniation } \\
(\mathrm{n} / \%)\end{array}$ & $55(42.6)$ & $32(58.2)$ & 0.867 & 0.355 & $5(22.7)$ & $38(45.2)$ & 3.664 & 0.056 \\
\hline $\begin{array}{l}\text { Decompressive } \\
\text { craniectomy } \\
(\mathrm{n} / \%)\end{array}$ & $46(35.7)$ & $22(25.0)$ & 2.762 & 0.097 & $9(40.9)$ & 33(39.3) & 0.019 & 0.890 \\
\hline Shock (n/\%) & $34(26.4)$ & $22(25.0)$ & 0.050 & 0.823 & $6(27.3)$ & $20(23.8)$ & 0.113 & 0.737 \\
\hline $\begin{array}{l}\text { The length of } \\
\text { NICU stay } \\
\text { (days) }\end{array}$ & $14 \pm 7$ & $12 \pm 6$ & 0.37 & 0.545 & $16 \pm 5$ & $16 \pm 7$ & 0.554 & 0.582 \\
\hline $\begin{array}{l}\text { GCS at transfer } \\
\text { out }\end{array}$ & $7 \pm 3$ & $7 \pm 2$ & 0.86 & 0.364 & $6 \pm 2$ & $7 \pm 3$ & 0.276 & 0.784 \\
\hline $\begin{array}{l}\text { NICU mortality } \\
(\mathrm{n} / \%)\end{array}$ & $11(8.5)$ & $6(6.8)$ & 0.212 & 0.646 & $1(4.5)$ & $2(2.4)$ & & 0.506 \\
\hline $\begin{array}{l}\text { The data are exp } \\
\text { not normally dis } \\
\text { acute physiology }\end{array}$ & $\begin{array}{l}\text { ied as t } \\
\text { ited. BN } \\
\text { d chron }\end{array}$ & $\begin{array}{l}\text { ean } \pm s \\
\text { dy ma } \\
\text { alth ev }\end{array}$ & $\begin{array}{l}\text { norm } \\
\text { dex; } \\
\text { tion I }\end{array}$ & 1 & $\begin{array}{l}\text { ed or as } \\
\text { V Coma S } \\
\text { T, isolates }\end{array}$ & $\begin{array}{l}\text { ian (inte } \\
\text {; APACH } \\
\text { tal deep }\end{array}$ & $\begin{array}{l}\text { uartile } \\
\text { II score } \\
\text { in thro }\end{array}$ & $\begin{array}{l}\text { ge) if } \\
\text { esis. }\end{array}$ \\
\hline
\end{tabular}


Table 3

Difference of demographic data, clinical data between IDDVT and proximal DVT groups

\begin{tabular}{|lllll|}
\hline Variables & IDDVT & proximal DVT & t/x & $p$ \\
\hline Age (years) & $\mathbf{n = 1 2 9}$ & $\mathbf{n = 2 8}$ & & \\
\hline Male (n/\%) & $62 \pm 12$ & $62 \pm 16$ & 0.194 & 0.847 \\
\hline BMI(kg/m²) & $65(50.4)$ & $10(35.7)$ & 1.783 & 0.182 \\
\hline GCS on admission & $21 \pm 7$ & $22 \pm 7$ & -0.731 & 0.469 \\
\hline APACHE II score & $6 \pm 2$ & $6 \pm 2$ & 0.401 & 0.691 \\
\hline Brain herniation (n/\%) & $23 \pm 3$ & $24 \pm 3$ & -1.577 & 0.123 \\
\hline Decompressive craniectomy (n/\%) & $55(42.6)$ & $8(28.6)$ & 1.894 & 0.169 \\
\hline Shock (n/\%) & $46(35.7)$ & $11(39.3)$ & 0.131 & 0.718 \\
\hline The length of NICU stay (days) & $34(26.4)$ & $8(28.6)$ & 0.058 & 0.810 \\
\hline GCS at transfer out & $14 \pm 7$ & $16 \pm 6$ & -1.410 & 0.166 \\
\hline NICU mortality & $7 \pm 3$ & $6 \pm 2$ & 1.025 & 0.310 \\
\hline $\begin{array}{l}\text { The data are expressed as the mean } \pm \text { SD if normally distributed or as median (interquartile range) if } \\
\text { not normally distributed. BMI, body mass index; GCS, Glasgow Coma Scale; APACHE II score, the } \\
\text { acute physiology and chronic health evaluation II score; DVT, deep vein thrombosis; IDDVT, isolated } \\
\text { distal deep vein thrombosis. }\end{array}$ & & & \\
\hline
\end{tabular}

Table 4

Multivariate analysis of patient-related risk factors

for IDDVT occurrence

\begin{tabular}{|lll|}
\hline Variables & \multicolumn{2}{c|}{ IDDVT occurrence } \\
\cline { 2 - 3 } & OR(95\% Cl) & $\boldsymbol{p}$ \\
\hline Age & $1.012-1.061$ & 0.003 \\
\hline GCS on admission & $0.672-0.925$ & 0.003 \\
\hline The length of NICU stay & $1.010-1.119$ & 0.020 \\
\hline BMI & $0.866-0.980$ & 0.10 \\
\hline BMI, body mass index; GCS, Glasgow coma scale. \\
\hline
\end{tabular}

Patients who developed IDDVT were older and had a higher percentage of female, a lower BMI, a higher incidence of cardiovascular disease, a lower GCS score at admission and longer surgery time than those 
who did not develop IDDVT. Multivariate logistic regression analysis showed that age, GCS score at admission, length of NICU stay and BMI were risk factors for the development of IDDVT.

Patients who developed IDDVT extension did not have significant difference in the baseline characteristics, disease characteristics, treatment approaches and patient outcomes compared with those who did not develop IDDVT extension. Similar results were shown on the comparisons of patients with IDDVT and patients with proximal DVT. Due to low sample size, multivariate logistic regression analysis did not show any risk factor for the extension of IDDVT.

Other Comparing results of disease history, comorbidities and treatment approaches are provided in the supplemental digital content.

\subsection{Dynamic changes in coagulation tests and their predictive values for IDDVT occurrence and extension} (Fig. 2-3)

APTT, D-dimer, TT, fibrinogen, and platelets showed significant differences within groups but not between groups depending on whether IDDVT occurred. PT did not differ between or within groups.

D-dimer and fibrinogen exhibited significant differences between and within groups according to whether IDDVT extension occurred. TT and platelets exhibited significant differences within groups but not between groups. PT and APTT did not differ between and within groups.

D-dimer levels during the NICU stay were sensitive predictors of IDDVT extension. The highest AUC value for the ROC analysis of D-dimer level was on days 5-7 (the area under the ROC was 0.89 , with a $95 \% \mathrm{Cl}$ of $0.82-0.95)$. Based on the Youden index, the cutoff point in the ROC analysis of D-dimer levels on days 5-7 for predicting IDDVT extension was set to $8.9 \mathrm{mg} / \mathrm{L}$, achieving a sensitivity and specificity of 0.86 and 0.81 , respectively.

Fibrinogen on days 5-7 was also a predictor of IDDVT extension. However, the area under the ROC of fibrinogen on days $5-7$ was significant smaller than that of D-dimer level on days $5-7$ ( 0.69 vs. $0.89, p=$ 0.0012). The increase in the D-dimer level on days 5-7 compared with day 1 of the NICU stay were also valuable for predicting IDDVT extension. However, the area under the ROC of the increase in the D-dimer level on days $5-7$ was smaller than that of $D$-dimer level on days $5-7$ ( 0.76 vs. $0.89, p=0.0022)$. Hence, D-dimer level on days $5-7$ is the most valuable predictor of IDDVT extension in our study.

The boxplots of dynamic changes in PT, APTT, fibrinogen, TT were showed in the supplemental digital content.

\section{Discussion}

The present study demonstrated that the occurrence and extension of IDDVT were common in ABI patients. Age, GCS score at admission, BMI and length of NICU stay were risk factors for the development of IDDVT. D-dimer level on days 5-7 may help to diagnose IDDVT extension. 
In this study, the incidence of IDDVT occurrence and extension in ABI patients in the NICU was high, reaching $61.6 \%$ and $20.8 \%$, respectively. These rates were higher than those reported in other studies [2]. The reason for the discrepancy may be due to the characteristics of the included patients. Most of our included patients were elderly people with severe $A B I$. The incidence rates of brain herniation and shock in our patients were $39 \%$ and $26 \%$, respectively. Because of disease severity, prolonged mechanical ventilation, deep analgesia and sedation, vasoactive drugs were commonly administered; these agents are risk factors for DVT occurrence $[13,14]$. Another factor that probably contributed to the high IDDVT incidence was the low rate and late initiation of anticoagulation [15]. Thirty-eight percent of the included patients received anticoagulants, which accounted for $52 \%$ of DVT patients. The median starting time for anticoagulation was the eighth day of the NICU stay. The aim of anticoagulation was treatment rather than prophylaxis. Actually, even after the DVT prophylaxis guideline in neurocritical care patients was published in 2016[16], the proportion of pharmacological VTE prophylaxis that was concordant with the guideline varied from 22.1 to $43.0 \%$ in a recent retrospective cohort study of 4632 neurocritical care patients[17]. Hence, further efforts should be made to narrow the gap between guideline-recommended care and current practice.

The risk factors for IDDVT occurrence found in our study, such as age, disease severity and length of NICU stay, are consistent with previous literature[18, 19]. Other potential risk factors for IDDVT occurrence, such as prolonged mechanical ventilation, deep analgesia and sedation, duration of surgery, and renal-replacement therapy, were not found to be risk factors in our study because of either common or nearly seldom application. However, no risk factors for IDDVT extension were found. Actually, no differences in patients' characteristics were revealed between patients with IDDVT extension and without and between patients with IDDVT and with proximal DVT. Furthermore, most of DVT patients were asymptomatic. It implies that distinguishing patients with IDDVT extension from those without IDDVT extension is difficulty. In our study, it seemed that IDDVT occurrence and extension did not lead to worse patients' outcomes. It may be because of the short observation period, focusing only on the NICU stay. More research is needed on the influence of IDDVT on patients' long term outcomes

In the current study, D-dimer levels in serial during the NICU stay were found to be a predictor of IDDVT extension in ABI patients, especially D-dimer on days 5-7. As in previous research, postoperative D-dimer was almost universally elevated beyond the standard threshold of $0.5 \mathrm{mg} / \mathrm{L}$ [20]. D-dimer is influenced by various factors, leading to its low value with single data to diagnose DVT in perioperative patients[21]. However, it was shown that D-dimer was valuable for diagnosing DVT with a higher threshold value in neurocritical patients [20,22,23], as well as for predicting recurrence risk of venous thromboembolism[24]. The pattern of D-dimer change showed that it peaked on days 5-7, which was two days before the most common time of IDDVT extension in our patients. Compared with other coagulation indices, such as D-dimer on days $2-4$ or $>7$, relative changes in D-dimer, and fibrinogen, Ddimer on days 5-7 was the most valuable predictor for IDDVT extension in our patients. More caution should be paid to $A B I$ patients with significant increase in $D$-dimer during perioperative period due to the high risk of IDDVT extension. 
Whether IDDVT patients should receive anticoagulation treatment is an unresolved problem. Existing evidence demonstrates that in low-risk outpatients, anticoagulation treatment may produce little or no decrease in the risk of IDDVT extension but increase the risk of bleeding [25, 26]. In these patients, ultrasound surveillance seems to be a priority for anticoagulant therapy. However, for high-risk IDDVT patients, for instance, the severe ABI patients in our study, the optimal management protocol has not been established, although anticoagulation treatment has been suggested according to the guidelines [7]. The higher proportion of patients with IDDVT extension receiving anticoagulants ( $98 \%$ vs. $49 \%$ ) did not decrease the risk of IDDVT extension. On the other hand, anticoagulation treatment in our study did not increase the risk of gastrointestinal bleeding or the need for transfusion or reoperation. In addition, the incidence of PE in our study was low $(0.6 \%)$. Hence, anticoagulation treatment ( $73 \%$ of patients with IDDVT extension receiving half of the dose of coagulant commonly used) seems to be safe in our patients, but its efficiency needs further research.

\section{Limitations}

Our study has several shortcomings. First, thrombophilia testing was not performed in the present study. However, the included ABI patients did not show characteristics associated with inherited thrombophilia [27]. Second, the observation period was short, focusing only on the NICU stay. The impact of IDDVT occurrence, extension and recurrence on $A B I$ patients' long-term outcomes needs to be studied further. Third, the proportion of $A B I$ varied greatly, ranging from $48 \%$ in acute hemorrhagic stroke to $15 \%$ in acute ischemic stroke. Fourth, DVT in the upper limbs was not considered in this study. Finally, the retrospective design of the study renders it susceptible to unknown confounding factors, and the sample size was small.

\section{Conclusion}

The occurrence and extension of IDDVT are common in ABI patients. Clinicians should be on the alert for IDDVT occurrence in patients who are elderly, severe injury, have more length of NICU stays. D-dimer level on days $5-7$ is the most valuable predictor of IDDVT extension in severe ABI patients.

\section{Abbreviations}

IDDVT, isolated distal deep vein thrombosis; $\mathrm{ABI}$, acute brain injury; NICU, neurocritical care unit; DVT, deep vein thrombosis; CCUS, Complete compression ultrasound; PT, prothrombin time; APTT activated partial thromboplastin time; TT, thrombin time; ROC, receiver operating characteristic.

\section{Declarations}

\section{Ethics approval and consent to participate}


The study was approved by the Ethics Committee of The First Affiliated Hospital of USTC (2021-RE-017). The requirement for informed consent was waived because of the minimal risk to participants.

\section{Consent for publication}

Not applicable.

\section{Availability of data and materials}

The datasets used and/or analyzed during the current study are available from the corresponding author on reasonable request.

\section{Competing interests}

The authors declare that they have no competing interests.

\section{Funding}

National Natural Science Foundation of China $(81870060,81871584)$

\section{Authors' contributions}

RT, ZG, MD, HYL, YYY, JZS and MZ conceived of the study, participated in its design and coordination and helped to draft the manuscript. RT wrote the manuscript. RT, ZG and MD collected the data and performed the statistical analysis. All authors critically reviewed, drafted and approved the manuscript for publication. All authors read and approved the final manuscript.

\section{Acknowledgements}

The authors acknowledge Mr. Ming Yang, Mrs. Xian Huang, and Mrs. Ting Chang for their professional skill in CCUS.

\section{References}

1. Skrifvars MB, Bailey M, Presneill J, French C, Nichol A, Little L, Duranteau J, Huet O, Haddad S, Arabi $Y$, et al. Venous thromboembolic events in critically ill traumatic brain injury patients. Intensive Care Med. 2017;43(3):419-28.

2. Zhang P, Bian Y, Xu F, Lian L, Zhu S, Tang Z, Wang F. The Incidence and Characteristics of Venous Thromboembolism in Neurocritical Care Patients: A Prospective Observational Study. Clin Appl Thromb Hemost. 2020;26:1-7.

3. Liang CW, Su K, Liu JJ, Dogan A, Hinson HE. Timing of deep vein thrombosis formation after aneurysmal subarachnoid hemorrhage. J Neurosurg. 2015;123(4):891-6.

4. Yang T, Wei G, Zhu C, Pan A. [Incidence and risk factor analysis of deep venous thrombosis in patients with severe traumatic brain injury]. Zhonghua wei zhong bing ji jiu yi xue. 2019;31(2):182-6. 
5. Palareti G. How I treat isolated distal deep vein thrombosis (IDDVT). Blood. 2014;123(12):1802-9.

6. Robert-Ebadi H, Righini M. Management of distal deep vein thrombosis. Thromb Res. 2017;149:4855 .

7. Kearon C, Akl EA, Ornelas J, Blaivas A, Jimenez D, Bounameaux H, Huisman M, King CS, Morris TA, Sood N, et al. Antithrombotic Therapy for VTE Disease: CHEST Guideline and Expert Panel Report. Chest. 2016;149(2):315-52.

8. Bernardi E, Camporese G, Büller HR, Siragusa S, Imberti D, Berchio A, Ghirarduzzi A, Verlato F, Anastasio R, Prati C, et al. Serial 2-point ultrasonography plus D-dimer vs whole-leg color-coded Doppler ultrasonography for diagnosing suspected symptomatic deep vein thrombosis: a randomized controlled trial. JAMA. 2008;300(14):1653-9.

9. Ageno W, Camporese G, Riva N, lotti M, Bucherini E, Righini M, Kamphuisen PW, Verhamme P, Douketis JD, Tonello C, et al. Analysis of an algorithm incorporating limited and whole-leg assessment of the deep venous system in symptomatic outpatients with suspected deep-vein thrombosis (PALLADIO): a prospective, multicentre, cohort study. Lancet Haematol. 2015;2(11):e474-80.

10. Goodacre S, Sampson F, Thomas S, van Beek E, Sutton A. Systematic review and meta-analysis of the diagnostic accuracy of ultrasonography for deep vein thrombosis. BMC Med Imaging. 2005;5:6.

11. Hargrove KL, Barthol CA, Allen S, Franco-Martinez C. Surveillance Ultrasound in the Neuro Intensive Care Unit: Time to Deep Vein Thrombosis Diagnosis. Neurocrit Care. 2019;30(3):645-51.

12. Needleman L, Cronan JJ, Lilly MP, Merli GJ, Adhikari S, Hertzberg BS, DeJong MR, Streiff MB, Meissner MH: Ultrasound for Lower Extremity Deep Venous Thrombosis: Multidisciplinary Recommendations From the Society of Radiologists in Ultrasound Consensus Conference. Circulation 2018, 137(14):1505-1515.

13. Ibrahim EH, Iregui M, Prentice D, Sherman G, Kollef MH, Shannon W. Deep vein thrombosis during prolonged mechanical ventilation despite prophylaxis. Crit Care Med. 2002;30(4):771-4.

14. Minet C, Potton L, Bonadona A, Hamidfar-Roy R, Somohano CA, Lugosi M, Cartier JC, Ferretti G, Schwebel C, Timsit JF. Venous thromboembolism in the ICU: main characteristics, diagnosis and thromboprophylaxis. Crit Care. 2015;19(1):287.

15. Abdel-Aziz H, Dunham CM, Malik RJ, Hileman BM. Timing for deep vein thrombosis chemoprophylaxis in traumatic brain injury: an evidence-based review. Crit Care. 2015;19(1):96.

16. Nyquist P, Bautista C, Jichici D, Burns J, Chhangani S, DeFilippis M, Goldenberg FD, Kim K, LiuDeRyke X, Mack W, et al. Prophylaxis of Venous Thrombosis in Neurocritical Care Patients: An Evidence-Based Guideline: A Statement for Healthcare Professionals from the Neurocritical Care Society. Neurocrit Care. 2016;24(1):47-60.

17. Sauro KM, Soo A, Kramer A, Couillard P, Kromm J, Zygun D, Niven DJ, Bagshaw SM, Stelfox HT. Venous Thromboembolism Prophylaxis in Neurocritical Care Patients: Are Current Practices, Best Practices? Neurocrit Care. 2019;30(2):355-63. 
18. Tritschler T, Kraaijpoel N, Le Gal G, Wells PS. Venous Thromboembolism: Advances in Diagnosis and Treatment. JAMA. 2018;320(15):1583-94.

19. Mazzolai L, Aboyans V, Ageno W, Agnelli G, Alatri A, Bauersachs R, Brekelmans MPA, Büller HR, Elias $A$, Farge $D$, et al. Diagnosis and management of acute deep vein thrombosis: a joint consensus document from the European Society of Cardiology working groups of aorta and peripheral vascular diseases and pulmonary circulation and right ventricular function. Eur Heart J. 2018;39(47):420818.

20. Prell J, Rachinger J, Smaczny R, Taute BM, Rampp S, Illert J, Koman G, Marquart C, Rachinger A, Simmermacher $\mathrm{S}$, et al. D-dimer plasma level: a reliable marker for venous thromboembolism after elective craniotomy. J Neurosurg. 2013;119(5):1340-6.

21. Bockenstedt P. D-dimer in venous thromboembolism. N Engl J Med. 2003;349(13):1203-4.

22. Kuwashiro T, Toyoda K, Oyama N, Kawase K, Okazaki S, Nagano K, Koga M, Matsuo H, Naritomi H, Minematsu K. High plasma D-dimer is a marker of deep vein thrombosis in acute stroke. J Stroke Cerebrovasc Dis. 2012;21(3):205-9.

23. Natsumeda M, Uzuka T, Watanabe J, Fukuda M, Akaiwa Y, Hanzawa K, Okada M, Oishi M, Fujii Y. High Incidence of Deep Vein Thrombosis in the Perioperative Period of Neurosurgical Patients. World Neurosurg. 2018;112:e103-12.

24. Kyrle PA, Eichinger S. Clinical scores to predict recurrence risk of venous thromboembolism. Thromb Haemost. 2012;108(6):1061-4.

25. Righini M, Galanaud JP, Guenneguez H, Brisot D, Diard A, Faisse P, Barrellier MT, Hamel-Desnos C, Jurus $\mathrm{C}$, Pichot $\mathrm{O}$, et al. Anticoagulant therapy for symptomatic calf deep vein thrombosis (CACTUS): a randomised, double-blind, placebo-controlled trial. Lancet Haematol. 2016;3(12):e556-62.

26. Utter GH, Dhillon TS, Salcedo ES, Shouldice DJ, Reynolds CL, Humphries MD, White RH. Therapeutic Anticoagulation for Isolated Calf Deep Vein Thrombosis. JAMA Surg. 2016;151(9):21.

27. Connors JM. Thrombophilia Testing and Venous Thrombosis. N Engl J Med. 2017;377(12):1177-87.

\section{Figures}




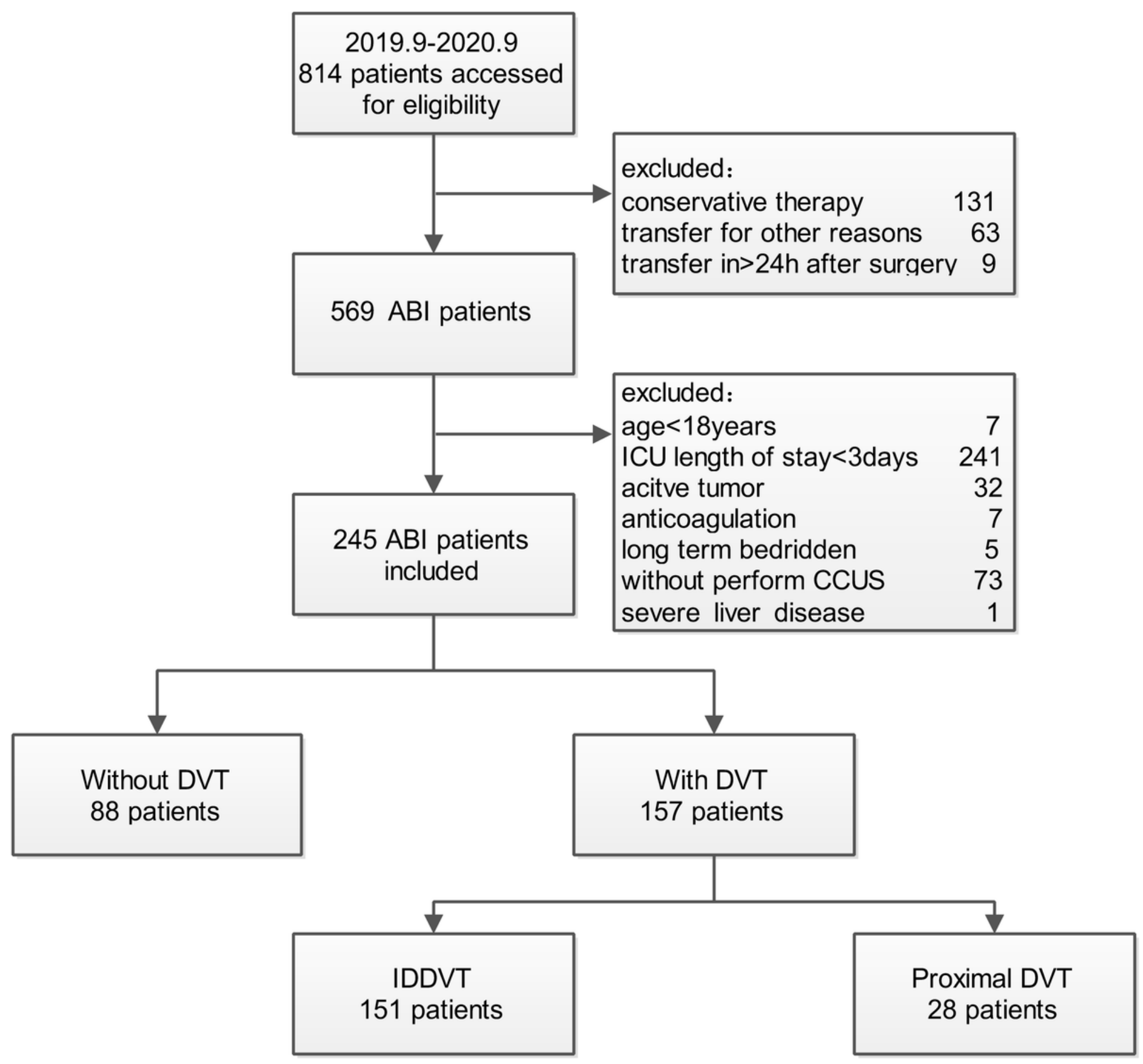

Figure 1

Flowchart outlining the inclusion of patients in this cohort study. 

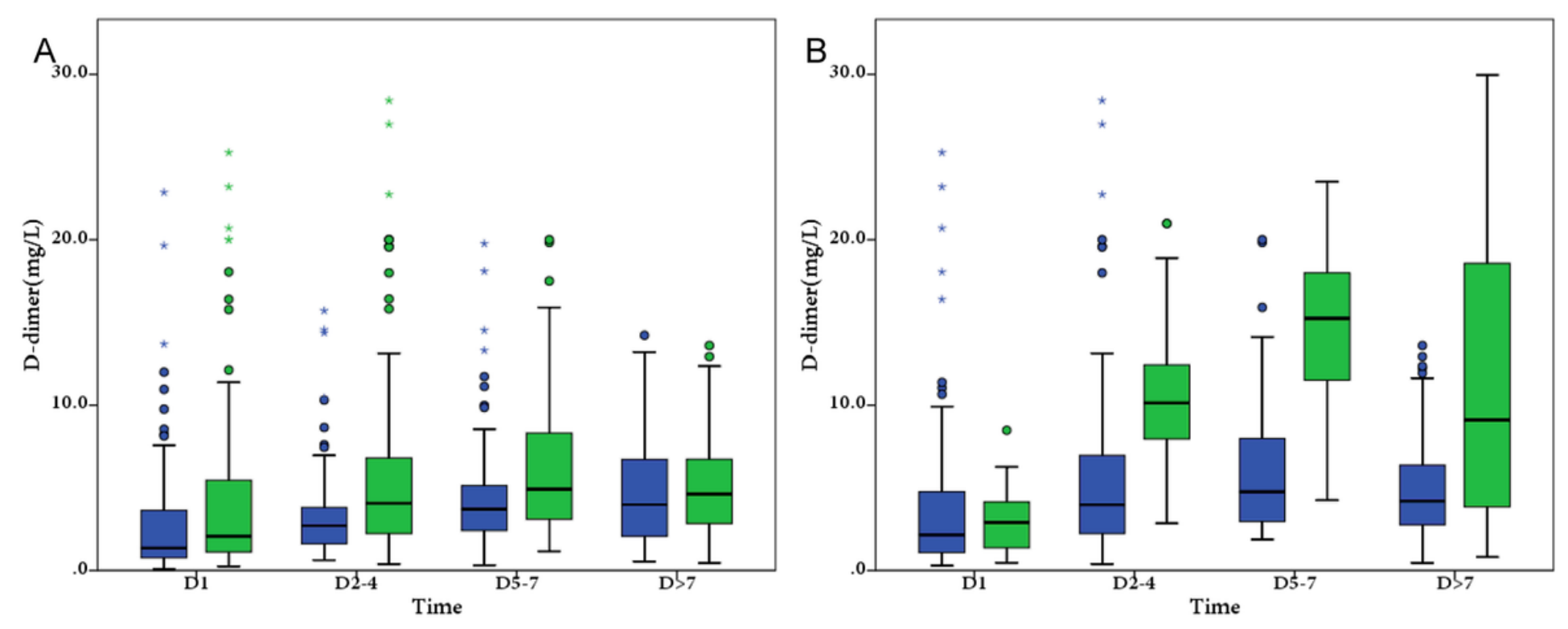

Figure 2

Dynamic changes of D-dimer among patients with IDDVT occurrence or extension compared with control subjects A, patients with IDDVT occurrence vs. without Green box, patients with IDDVT occurrence (n =79); blue box, patients without IDDVT occurrence $(n=45) . p=0.083$ for the effect of IDDVT occurrence or not; $p=0.032$ for the effect of dynamic changes; $p=0.415$ for interaction. $B$, patients with IDDVT extension vs. without Green box, patients with IDDVT extension $(n=22)$; blue box, patients without IDDVT extension $(n=84)$. $p<0.0001$ for the effect of IDDVT extension or not; $p<0.0001$ for the effect of dynamic changes; $p<0.0001$ for interaction. 


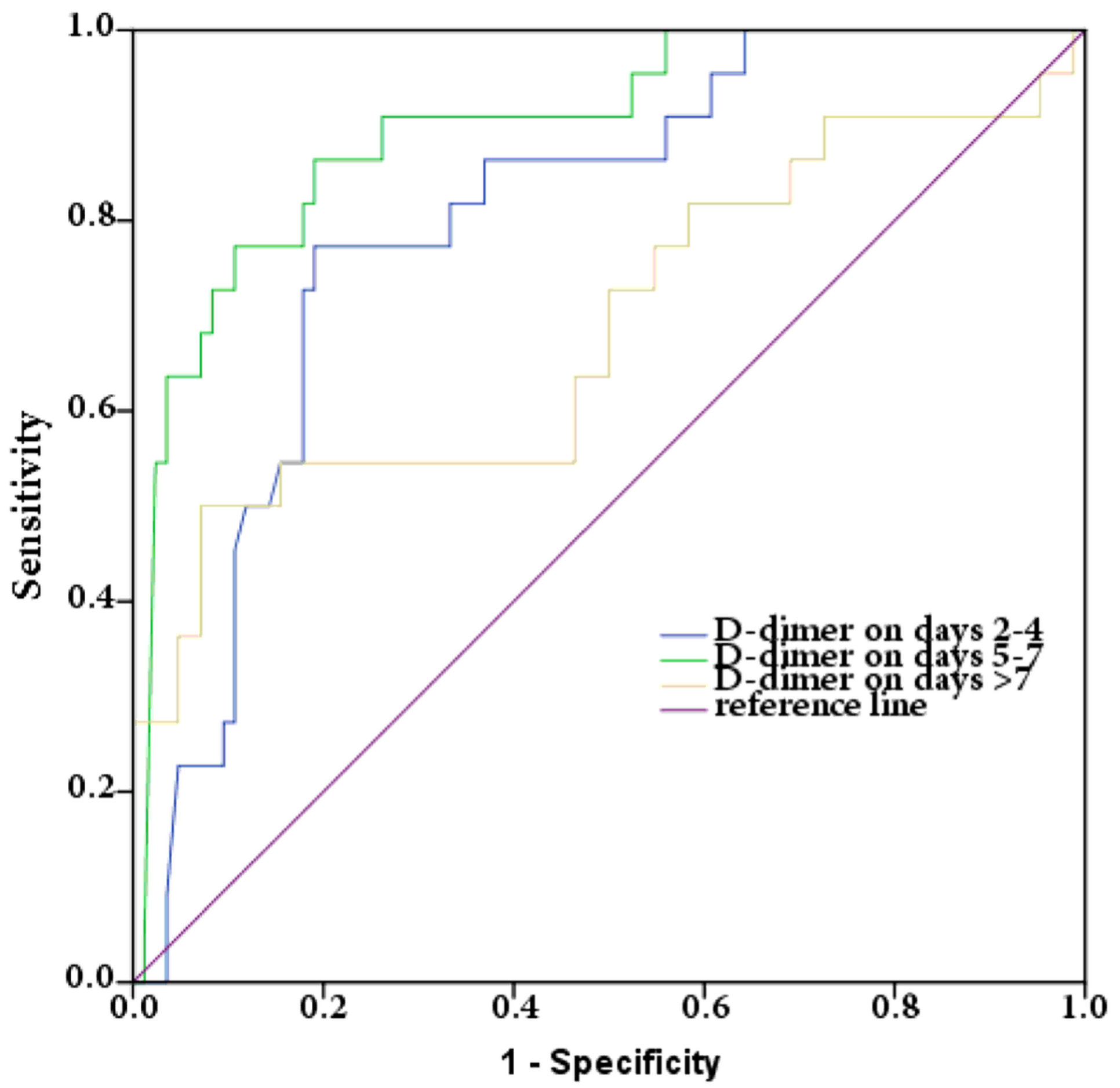

Figure 3

Receiver operating characteristic curve of serial D-dimer results to diagnose IDDVT extension The area under the ROC of D-dimer on days 2 - 4 was 0.80 , with a $95 \% \mathrm{Cl}$ of $0.71-0.87$; the area under the ROC of D-dimer on days $5-7$ was 0.89 , with a $95 \% \mathrm{Cl}$ of $0.82-0.95$; the area under the ROC of D-dimer on days $>7$ was 0.69 , with a $95 \% \mathrm{Cl}$ of $0.59-0.77$.

\section{Supplementary Files}


This is a list of supplementary files associated with this preprint. Click to download.

- supple.docx 